УДК 591.111.1

\title{
Detection of the State of Thrombotic Readiness in Rats under One-Time Suprathreshold Physical Activity of Various Durations by Means of Thromboelastography
}

\author{
Alexander A. Blazhko*a, \\ Igor I. Shakhmatov ${ }^{\mathrm{a}, \mathrm{b}}$, Igor V. Kovalev ${ }^{\mathrm{c}}$, \\ Valeriy I. Kiselev ${ }^{\mathrm{a}, \mathrm{b}}$, Yuliya A. Bondarchuk ${ }^{\mathrm{a}, \mathrm{b}}$, \\ Oksana M. Ulitina ${ }^{a, b}$ and Olga V. Alekseyeva ${ }^{a}$ \\ ${ }^{a}$ Altai State Medical University of the Ministry \\ of Health of the Russian Federation \\ 40 Lenin, Barnaul, 656038, Russia \\ ${ }^{b}$ Research Institute of Physiology \\ and Fundamental Medicine of SB RAS \\ 4 Timakov Str., Novosibirsk, 630117, Russia \\ 'Siberian State Medical University \\ 2 Moskovsky Trakt, Tomsk, 634050, Russia
}

Received 27.11.2017, received in revised form 15.04.2018, accepted 18.06.2018

Suprathreshold physical activity causing distress in the organism can lead to the damage of various organs and systems including the hemostatic system. A modern integrated method of the hemostatic system assessment is thromboelastography. The purpose of the present study was to evaluate the state of the hemostatic system under one-time suprathreshold physical activity of various durations by means of thromboelastography. Experimental groups of rats were exposed to 4-hour and 8-hour physical activities in the form of forced running on a moving platform with the speed of 6-8 $\mathrm{m} / \mathrm{min}$. Immediately after the one-time physical activity, blood samples taken from rats were examined using the thrombelastograph in the Natem mode for 35 minutes. The 4-hour physical activity caused a reduction in coagulation time (CT) and an increase in the alpha angle and the maximum clot firmness $(M C F)$. After the 8-hour activity, the thrombelastograph registered a reduction in coagulation time (CT), an increase in the alpha angle, a decrease in the clot formation time (CFT), a decrease in the maximum clot firmness (MCF), and a reduction in the maximum clot lysis (ML). The 4-hour physical activity resulted in partial activation of the hemostatic system without changing the fibrinolytic activity of blood plasm. The changes revealed in thromboelastography parameters indicate a high risk of the

(C) Siberian Federal University. All rights reserved

This work is licensed under a Creative Commons Attribution-NonCommercial 4.0 International License (CC BY-NC 4.0).

* Corresponding author E-mail address: blazhko_1990@mail.ru

ORCID: 0000-0003-2578-6858 (Blazhko A.); 0000-0002-0979-8560 (Shakhmatov I.); 0000-0002-2661-5965 (Bondarchuk Yu.) 
development of thrombotic readiness. The 8-hour physical activity causes a shift of the hemostatic system parameters in rats towards the increased clot formation: hypercoagulation, fibrinogen and platelet consumption, inhibition of fibrinolysis. The combination of changes in thromboelastogram parameters is indicative of the development of thrombotic readiness.

Keywords: physical activity, thromboelastography, thrombotic state.

Citation: Blazhko A.A., Shakhmatov I.I., Kovalev I.V., Kiselev V.I., Bondarchuk Yu.A., Ulitina O.M., Alekseyeva O.V. Detection of the state of thrombotic readiness in rats under one-time suprathreshold physical activity of various durations by means of thromboelastography. J. Sib. Fed. Univ. Biol., 2019, 12(4), 460-469. DOI: 10.17516/1997-1389-0312

\title{
Выявление состояния тромботической готовности у крыс при однократной сверхпороговой физической нагрузке разной продолжительности методом тромбоэластографии
}

\author{
А.А. Блажко ${ }^{\mathrm{a}}$ И.И. Шахматов ${ }^{\mathrm{a}, \boldsymbol{0}}$, \\ И.В. Ковалев ${ }^{\text {в }}$ В.И. Киселев ${ }^{\mathrm{a}, \boldsymbol{0}}$, \\ Ю.А. Бондарчук $\kappa^{\mathrm{a}, \tilde{0}}$, О.М. Улитина ${ }^{\mathrm{a}, \boldsymbol{0}}$, О.В. Алексеева ${ }^{\mathrm{a}}$ \\ алттайский государственный медицинский университет \\ Минздрава России \\ Россия, 656038, Барнаул, пр. Ленина, 40 \\ ${ }^{6}$ НИИ физиологии и фундаментальной медицины СО РАН \\ Россия, 630117, Новосибирск, ул. Тимакова, 4 \\ ${ }^{8}$ Сибирский государственный медицинский университет \\ Россия, 634050, Томск, тр. Московский, 2
}

Сверхпороговая физическая нагрузка, вызывая в организме состояние дистресса, может приводить к повреждению различных органов и систем, включая систему гемостаза. Актуальным интегральным методом оценки системы гемостаза является тромбоэластография. Целью исследования было оценить состояние системы гемостаза крыс при однократной сверхпороговой физической нагрузке разной продолжительности с использованием метода тромбоэластографии. Экспериментальные группы крыс подвергали четырехчасовой и восьмичасовой физической нагрузке в виде навязанного бега в тредбане со скоростью вращения 6-8 м/мин. Сразу после однократного воздействия физической нагрузкой кровь исследовали на тромбоэластографе в режиме Nateт в течение 35 мин. Четырехчасовая физическая нагрузка вызывала укорочение времени коагулячии (СТ), увеличение «угла альфа», а также повымение максимальной твердости сгустка (МСF). По истечении восьмичасовой физической нагрузки на тромбоэластограмме регистрировали укорочение времени коагуляции (CT), повышение показателя «угол альфа», отмечали сокращение времени формирования 
сгустка (CFT), уменьшение максимальной твердости сгустка (МСF) и снижениемаксимального лизиса сгустка (ML). Четырехчасовая физическая нагрузка у крыс вызывает частичную активацию системы гемостаза без изменения фибринолитической активности плазмы крови. Выявленные изменения параметров тромбоэластограммы указывают на высокую степень риска развития состояния тромботической готовности. Восьмичасовая физическая нагрузка вызывает смещуение показателей системы гемостаза у крыс в сторону усиления тромбообразования: гиперкоагулящия, потребление фибриногена и тромбоцитов, угнетение фибринолиза. Совокупность выявленных изменений параметров тромбоэластограммы соответствует развитию состояния тромботической готовности.

Ключевые слова: физическая нагрузка, тромбоэластография, состояние тромботической готовности.

Цитирование: Блажко, А.А. Выявление состояния тромботической готовности у крыс при однократной сверхпороговой физической нагрузке разной продолжительности методом тромбоэластографии / А.А. Блажко, И.И. Шахматов, И.В. Ковалев, В.И. Киселев, Ю.А. Бондарчук, О.М. Улитина, О.В. Алексеева // Журн. Сиб. федер. ун-т. Биология, 2019. 12(4). C. 460-469. DOI: 10.17516/1997-1389-0312

\section{Введение}

Физическая нагрузка является одним из наиболее распространенных видов стрессорного воздействия на человека и при регулярном действии на организм может повышать его устойчивость за счет механизмов перекрестной адаптации (Агаджанян и др., 2000; Пшенникова, 2001). Однако сверхпороговая физическая нагрузка может приводить к повреждению различных органов и систем, вызывая в организме состояние дистресса (Шахматов, 2010). Показано, что значительные физические нагрузки связаны с повышением риска возникновения сердечно-сосудистых патологий, в том числе внезапной сердечной смерти (Marijon et al., 2015), поэтому в настоящее время активно исследуется их влияние на состояние организма.

Система гемостаза признана одной из наиболее реактивных систем организма, играя важную роль в процессах адаптации (Баркаган, Момот, 2008), и при действии чрезмерных стрессоров различной природы может отвечать формированием состоя- ния тромботической готовности (Шахматов, 2010; Момот, 2011). Существует множество методик для определения показателей различных звеньев системы гемостаза, однако общую картину свертывания от начала коагуляции до процесса фибринолиза с определением вязкоупругих свойств сгустка в цельной крови позволяет зафиксировать интегральный метод - тромбоэластография (Trzebicki et al., 2009; Theusinger et al., 2011). В настоящее время метод тромбоэластографии широко используется как в клинической практике (Johansson et al., 2009; Рыжков и др., 2014), так и в рамках экспериментальных исследований (Peng, Rhind, 2015).

Исследованию изменений со стороны системы гемостаза при физической нагрузке разной продолжительности посвящено множество работ как отечественных, так и зарубежных авторов. Однако в них использованы методы для определения того или иного звена системы гемостаза плазмы крови; причем многие из этих методик коагулограммы сегодня устарели и не рекомендуются для 
исследований (Момот, 2011; Буланов, 2015). Мы же предлагаем рассмотреть данную проблему в совокупности всех звеньев системы гемостаза в цельной крови интегральным методом.

Целью исследования было оценить состояние системы гемостаза крыс при однократной сверхпороговой физической нагрузке разной продолжительности с использованием метода тромбоэластографии.

\section{Материалы и методы}

Исследования были выполнены на 30 крысах-самцах линии Wistar массой $250 \pm 20$ г. Животные были разделены на три группы (интактная и две экспериментальные).

Экспериментальные животные подвергались 4- и 8-часовой физической нагрузке в виде навязанного бега в тредбане со скоростью вращения 6-8 м/мин. Сразу после окончания воздействия физической нагрузки из печеночного синуса под эфирным наркозом забирали кровь для исследования в объеме 5 мл в полистироловый шприц с широкой иглой, содержащий 3,8\%-ный раствор цитрата натрия (соотношение крови и цитрата 9:1).

Запись тромбоэластограммы проводили на аппарате «RotemGamma» (Германия) в режиме Natem в течение 35 мин с использованием активатора «Star-tem». Оценивались следующие параметры тромбоэластограммы:

- Время коагуляции (СТ) - время от момента внесения реагента до достижения тромбоэластограммой амплитуды в 2 мм; данный интервал отражает фазу инициации свертывания крови (Kawassaki et al., 2004).

- Время формирования сгустка (СFT) время изменения амплитуды тромбоэластограммы с 2 до 20 мм; данный показатель характеризует фазу усиления процесса тромбообразования (Kawassaki et al., 2004).

- Угол альфа (ALP) - угол, образованный продольной осью тромбоэластограммы и прямой, проведенной по касательной к тромбоэластограмме из точки, соответствующей амплитуде сгустка 2 мм. Эта величина отражает кинетику образования сгустка и характеризует фазу распространения (Johansson et al., 2008).

- Максимальная твердость сгустка (MCF) - показатель, соответствующий максимальной амплитуде сгустка и отражающий функции тромбоцитов и фибриногена (Лычева и др., 2014).

- Максимальный лизис (ML) - показатель, характеризующий уровень максимального фибринолиза, зарегистрированного в течение анализа. Определяется как нахождение самой низкой амплитуды после достижения MCF.

Эксперименты с крысами выполняли в соответствии с Европейской конвенцией по охране позвоночных животных, используемых в эксперименте, а также Директивой 86/609/ЕЕС. Обезболивание животных проводили в соответствии с «Правилами проведения работ с использованием экспериментальных животных» (Приказ МЗ СССР № 775 от 12.08.1977 г.).

Полученные в ходе исследования данные представлены в таблице в виде (m [25-75 \%]), где $\mathrm{m}$ - медиана в выборочной совокупности; [25-75\%] - 25-й и 75-й перцентиль. Статистический анализ проводили с применением пакета прикладных программ Statistica 6.0 (StatSoft, США). Достоверность различий оценивали при помощи непараметрического U-критерия Манна - Уитни, так как признаки не подчинялись нормальному распределению. Различия считали достоверными при уровне статистической значимости $\mathrm{p}<0,05$. 


\section{Результаты и обсуждение}

Четырехчасовая физическая нагрузка вызывала активацию системы гемостаза у крыс, что проявлялось на тромбоэластограмме укорочением времени коагуляции (CT) на $25 \%(\mathrm{p}=0,031)$ и увеличением угла альфа на $6 \%(p=0,019)$ по сравнению с интактными животными (табл., рис. 1, 2). Однако время формирования сгустка (CFT) у экспериментальных животных, подвергшихся 4-часовой физической нагрузке, достоверно не отличалось от такового у интактных крыс $(\mathrm{p}=0,067)$. Исходя из этого, можно предположить, что 4-часовая физическая нагрузка вызывает лишь частичную активацию системы гемостаза у крыс, ускоряя наступление фазы инициации свертывания крови. Именно конечный результат фазы инициации процесса тромбообразования - активация функций тромбоцитов (Буланов и др., 2012) - является одной из реакций системы гемостаза в ответ на длительную физическую нагрузку (Hanke et al., 2010; Peat et al., 2010).

Максимальная твердость сгустка (MCF) после 4-часовой физической нагрузки у крыс повышалась на 9 \% ( $=0,006)$ по сравнению с интактными животными, что может быть рассмотрено как состояние гиперкоагуляции (Гриневич, 2010), повышение концентрации фибриногена и активности фактора XIII (Авдушкина и др., 2009; Лычева и др., 2014). Максимальный лизис сгустка (ML) после 4-часовой нагрузки достоверно не отличался от показателя интактных крыс.

По истечении 8-часовой физической нагрузки (рис. 3) на тромбоэластограмме регистрировалось укорочение времени коагуляции (СТ) на $26 \%$ (p =0,002) и повышение показателя «угол альфа» на $10 \%(\mathrm{p}<0,001)$ по сравнению с интактными животными. Также

Таблица. Показатели тромбоэластограммы интактных животных и животных сразу после 4-часовой и 8-часовой физической нагрузки

Table. Parameters of thromboelastogram of intact animals and animals immediately after 4-hour and 8-hour physical activity

\begin{tabular}{|c|c|c|c|}
\hline Показатели & $\begin{array}{c}\text { Интактные крысы } \\
\text { (n=10) }\end{array}$ & $\begin{array}{c}\text { 4-часовая физическая } \\
\text { нагрузка }(\mathrm{n}=10)\end{array}$ & $\begin{array}{c}\text { 8-часовая физическая } \\
\text { нагрузка }(\mathrm{n}=10)\end{array}$ \\
\hline $\begin{array}{l}\text { Время коагуляции (CT), } \\
\text { с }\end{array}$ & $\begin{array}{l}193,0 \\
{[167,3-206,3]}\end{array}$ & $\begin{array}{l}144,0[102,8-188,8] \\
\mathrm{p} 1=0,031(\Delta 1-25 \%)\end{array}$ & $\begin{array}{l}143,0[114,0-163,0] \\
\text { p1 }=0,002(\Delta 1-26 \%) \\
\text { p2 }=0,534\end{array}$ \\
\hline $\begin{array}{l}\text { Время формирования } \\
\text { сгустка (CFT), с }\end{array}$ & $\begin{array}{l}76,0 \\
{[64,3-106,5]}\end{array}$ & $\begin{array}{l}62,0[42,5-78,8] \\
\text { p1 }=0,067\end{array}$ & $\begin{array}{l}59,5[52,5-67,8] \\
\text { p1 }=0,028(\Delta 1-22 \%) \\
\text { p2 }=0,967\end{array}$ \\
\hline Угол альфа (ALP), ${ }^{0}$ & \begin{tabular}{|l}
73,0 \\
{$[68,5-77,0]$}
\end{tabular} & $\begin{array}{l}77,5[74,3-81,0] \\
\mathrm{p} 1=0,019(\Delta 1+6 \%)\end{array}$ & $\begin{array}{l}80,0[79,5-82,0] \\
\text { p1 }<0,001(\Delta 1+10 \%) \\
\text { p2 }=0,477\end{array}$ \\
\hline $\begin{array}{l}\text { Максимальная } \\
\text { твердость сгустка } \\
(\mathrm{MCF}), \text { мм }\end{array}$ & $\begin{array}{l}68,0 \\
{[62,0-70,8]}\end{array}$ & $\begin{array}{l}74,0[70,5-76,8] \\
\mathrm{p} 1=0,006(\Delta 1+9 \%)\end{array}$ & $\begin{array}{l}60,5[59,0-65,3] \\
\text { p1 }=0,018(\Delta 1-11 \%) \\
\text { p2 }<0,001(\Delta 1-18 \%)\end{array}$ \\
\hline $\begin{array}{l}\text { Максимальный лизис } \\
(\mathrm{ML}), \%\end{array}$ & $\begin{array}{l}4,0 \\
{[1,0-6,0]}\end{array}$ & $\begin{array}{l}9,5[0,3-20,8] \\
\mathrm{p} 1=0,482\end{array}$ & $\begin{array}{l}0,0[0,0-1,0] \\
\text { p1 }=0,013(\Delta 1-100 \%) \\
\text { p2 }=0,086\end{array}$ \\
\hline
\end{tabular}

Примечания: $\Delta 1$ - статистически значимая разница экспериментальной группы с интактными животными при $\mathrm{p}<0,05 ; \mathrm{p} 1$ - уровень значимости различий экспериментальной группы с интактными животными; $\Delta 2$ - статистически значимая разница экспериментальных групп между собой при $\mathrm{p}<0,05 ;$ р2 - уровень значимости различий экспериментальных групп между собой; $\mathrm{n}$ - количество животных в группе. 


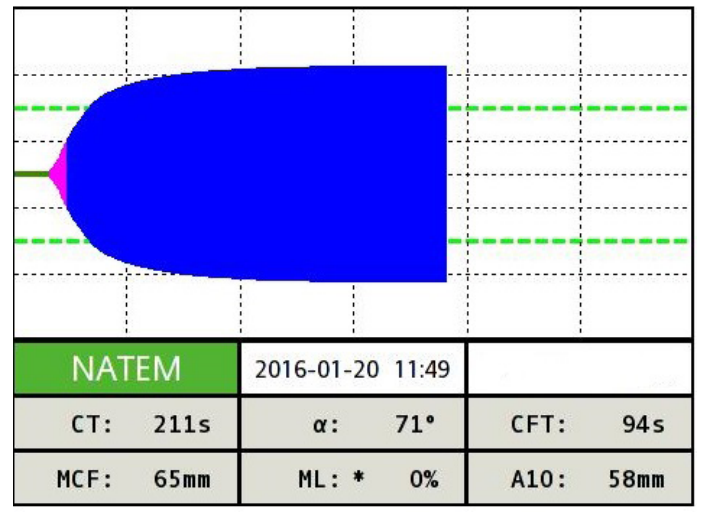

Рис. 1. Тромбоэластограмма интактной крысы. СТ - время коагуляции (c); $\alpha$ - угол альфа $\left({ }^{0}\right)$; CFT - время формирования сгустка (c); MCF - максимальная твердость сгустка (мм); ML - максимальный лизис (\%); А10 - амплитуда тромбоэластограмм на 10-й минуте теста

Fig. 1. Thromboelastogram of intact rat. CT - coagulation time $(\mathrm{s}) ; \alpha-$ alpha angle $\left({ }^{0}\right)$; CFT - clot formation time (s); MCF - maximum firmness of the clot (mm); ML - maximum lysis (\%) and A10 - amplitude of thromboelastograms in a 10 minute test

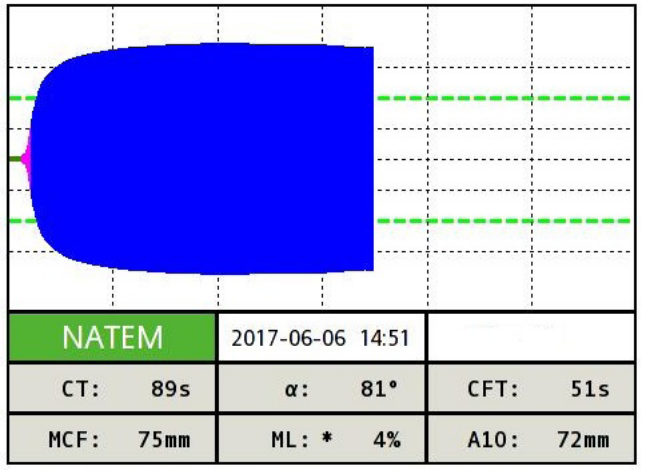

Рис. 2. Тромбоэластограмма крысыпосле 4-часовой физической нагрузки. Обозначения как на рис. 1

Fig. 2. Thromboelastogram of a rat after 4 hours of physical activity. See notations for Fig. 1

после 8-часовой нагрузки впервые отмечалось укорочение времени формирования сгустка (CFT) на $22 \%(p=0,028)$ по сравнению с показателями интактных животных. Это говорит о сдвиге системы гемостаза в сторону гиперкоагуляции, характеризующейся активацией как плазменных факторов свертывания, так и тромбоцитов.

Максимальная твердость сгустка (MCF) по завершении 8-часового стрессорного воздействия уменьшалась на $11 \%(\mathrm{p}=0,018)$ по

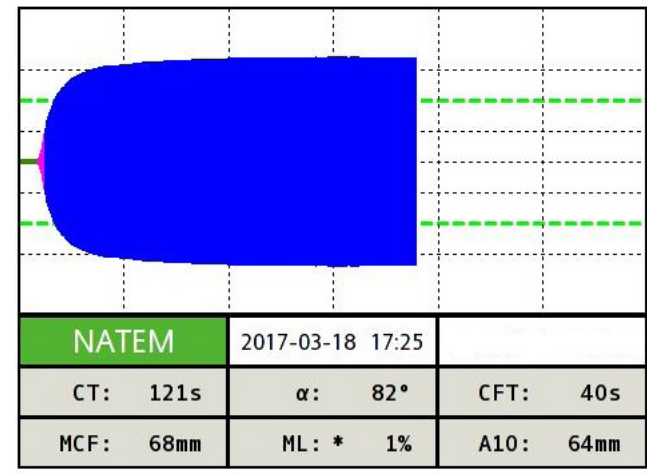

Рис. 3. Тромбоэластограмма крысы после 8-часовой физической нагрузки. Обозначения как на рис. 1

Fig. 3. Thromboelastogram of a rat after 8 hours of physical activity. See notations for Fig. 1

сравнению с интактными животными и на $18 \%(\mathrm{p}<0,001)$ относительно аналогичного параметра у крыс, подвергавшихся 4-часовой нагрузке. Изменение данного показателя может объясняться уменьшением количества тромбоцитов и снижением уровня фибриногена в крови экспериментальных животных вследствие их потребления на внутрисосудистое тромбообразование, что было установлено ранее (Блажко и др., 2016). Максимальный лизис сгустка (ML) после 8-часовой нагрузки 
в отличие от интактных животных не определялся, что может свидетельствовать о снижении фибринолитической активности плазмы крови. Данные же зарубежных авторов (Sumann et al., 2007; Sucker et al., 2010) говорят об активации процессов фибринолиза после длительных физических нагрузок у человека, в научных исследованиях отмечалось увеличение количества тканевого активатора плазминогена (t-PA) на фоне снижения печеночного клиренса t-PA (Fras et al., 2004).

Однако мы предполагаем, что предложенная нами модель 8-часовой физической нагрузки у крыс является более интенсивной, чем в представленных исследованиях, и вызывает в организме развитие дисфункции эндотелия с повышением выделения ингибитора активации плазминогена (PAI-1) и уменьшением количества тканевого активатора плазминогена (t-PA) (Мельникова, Макарова, 2015), что и обуславливает угнетение процесса фибринолиза. Уменьшение активности тканевого активатора плазминогена (t-PA), возможно, связано с уменьшением выработки эндотелиального оксида азота при развитии эндотелиальной дисфункции (Stamler, 1994), которая была выявлена нами ранее у крыс после 8-часовой физической нагрузки (Блажко и др., 2017).

Снижение фибринолитической активности плазмы крови после сверхпороговой физической нагрузки ранее было выявлено нами методом спонтанного эуглобулинового фибринолиза сгустка (Шахматов, 2010; Блажко и др., 2016) и подтверждено в данном исследовании более современным интегральным методом.

\section{Заключение}

4-часовая физическая нагрузка у крыс вызывает частичную активацию системы гемостаза без изменения фибринолитической активности плазмы крови. Выявленные изменения параметров тромбоэластограммы указывают на высокую степень риска развития состояния тромботической готовности. Однако мы предполагаем, что данная гемостазиологическая картина еще соответствует состоянию адаптации или эустрессу, когда фибринолитическая система и антикоагулянтная активность плазмы сдерживают нарастающую гиперкоагуляцию.

8-часовая физическая нагрузка вызывает смещение показателей системы гемостаза у крыс в сторону усиления тромбообразования: гиперкоагуляция, потребление фибриногена и тромбоцитов, угнетение фибринолиза. Совокупность выявленных изменений параметров тромбоэластограммы соответствует развитию состояния тромботической готовности. Состояние тромботической готовности служит подтверждением развития дистресса, или срыва адаптации, у экспериментальных животных со стороны системы гемостаза.

В дальнейшем необходимо изучение влияния сверхпороговой физической нагрузки на состояние системы гемостаза другими современными методами, такими как тромбодинамика, тест генерации тромбина и так далее, и возможное изучение молекулярных механизмов полученных изменений. Также представляется актуальным изучение способов корректировки гемостазиологической картины, возникающей при сверхпороговой физической нагрузке.

\section{Благодарности / Acknowledgements}

Исследования выполнены на средства в рамках проекта «Грант ректора АГМУ», договор № 1-гр от 21.02.2017.

The research was supported by "Grant of the Rector of ASMU”, contract No. 1-gr of 21.02.2017.

$$
-466-
$$




\section{Список литературы / References}

Авдушкина Л.А., Вавилова Т.В., Зыбина Н.Н. (2009) Метод тромбоэластографии/тромбоэластометрии в оценке системы гемостаза: прошлое и настоящее. Референсные интервалы. Клинико-лабораторный консилиум, 5: 26-33 [Avdushkina L.A., Vavilova T.V., Zybina N.N. (2009) Thrombelastography / thromboelastometry in the estimation of hemostasis system: the past and the present. Reference ranges. Clinical-Laboratory Concilium [Kliniko-laboratornyj konsilium], 5: 26-33 (in Russian)]

Агаджанян Н.А., Баевский Р.М., Береснева А.П. (2000) Учение о здоровье и проблемы адаптаичи. Ставрополь, Изд-во СГУ, 203 с. [Agadzhanyan N.A., Bayevsky R.M., Beresneva A.P. (2000) A study of health and adaptation problems. Stavropol, Stavropol State University, 203 p. (in Russian)]

Баркаган 3.С., Момот А.П. (2008) Диагностика и контролируемая терапия нарушенийгемостаза. M., 292 c. [Barkagan Z.S., Momot A.P. (2008) Diagnosis and controlled therapy of hemostatic disorders. Moscow, 292 p. (in Russian)]

Блажко А.А., Шахматов И.И., Лычева Н.А., Москаленко С.В. (2016) Снижение риска развития состояния тромботической готовности при воздействии сверхпороговой физической нагрузки у крыс на фоне предварительного приема пантогематогена. Современные проблемь науки и образования, 2 [Blazhko A.A., Shakhmatov I.I., Lycheva N.A., Moskalenko S.V. (2016) Risk reduction of thrombotic state of readiness under the influence of suprathreshold physical load in rats on the background of the anticipatory administration of pantogematogen. Modern Problems of Science and Education [Sovremennye problemy nauki i obrazovaniya], 2 (in Russian)]

Блажко А.А., Шахматов И.И., Киселев В.И., Лычева Н.А., Москаленко С.В. (2017) Изменения микроциркуляции у крыс по данным лазерной допплеровской флоуметрии при физической нагрузке, сопровождающейся развитием состояния тромботической готовности. Регионарное кровообращение и микрочиркуляция, 16(4): 60-64 [Blazhko A.A., Shakhmatov I.I., Kiselev V.I., Lycheva N.A., Moskalenko S.V. (2017) Changes of microcirculation in rats detected by laser Doppler flowmetry at physical load accompanied by the development of thrombotic readiness. Regional blood circulation and microcirculation [Regionarnoe krovoobrashchenie i mikrocirkulyaciya], 16(4): 60-64 (in Russian)]

Буланов А.Ю., Городецкий В.М., Щербакова О.В., Рязанова И.Б., Судейкина Н.Н., Шулутко Е.М., Орел Е.Б., Васильев С.А., Данишян К.И., Гржимоловский А.В., Карпов Е.Е., Полянская Т.Ю. (2012) Тромбоэластографическая оценка системы гемостаза и эффективности ее коррекции перед оперативными вмешательствами у больных с заболеваниями системы крови. Гематология и трансфузиология, 57(5): 36-42 [Bulanov A.Yu., Gorodetsky V.M., Shcherbakova O.V., Ryazanova I.B., Sudeikina N.N., Shulutko E.M., Orel E.B., Vasilyev S.A., Danishyan K.I., Grzhimalovsky A.V., Karpov E.E., Polyanskaya T.Yu. (2012) Thromboelastographic evaluation of the hemostasis system and efficiency of its correction before surgical interventions in hematological patients. Russian Journal of Hematology and Transfusiology [Gematologiya $\mathrm{i}$ transfuziologiya], 57(5): 36-42 (in Russian)]

Буланов А.Ю. (2015) Тромбоэластография в современной клинической практике. Аmлас ТЭГ. М., Ньюдиамед, 56 с. [Bulanov A.Yu. (2015) Thromboelastography in modern clinical practice. Atlas of TEG. Moscow, Nyudiamed, 56 p. (in Russian)]

$$
-467-
$$


Гриневич T.H. (2010) Ротационная тромбоэластометрия ROTEM как новый перспективный метод оценки системы гемостаза у пациентов травматологического профиля. Новости хирургии, 18(2): 115-122 [Grinevich T.N. (2010) Rotation thromboelastometry ROTEM as a new perspective method of the hemostasis system evaluation in patients of the traumatological profile. Surgery News [Novosti Khirurgii], 18(2): 115-122 (in Russian)]

Лычева Н.А., Шахматов И.И., Киселев В.И., Вдовин В.М. (2014) Изучение отставленного влияния гипотермии на параметры системы гемостаза у крыс. Бюллетень Сибирского отделения Российской академии медиџинских наук, 34(4): 25-29 [Lycheva N.A., Shakhmatov I.I., Kiselev V.I., Vdovin V.M. (2014) Investigation of hypothermia delayed influence on rats hemostatic system parameters. The Bulletin of Siberian Branch of Russian Academy of Medical Sciences [Byulleten' Sibirskogo otdeleniya Rossijskoj akademii medicinskih nauk], 34(4): 25-29 (in Russian)]

Мельникова Ю.С., Макарова Т.П. (2015) Эндотелиальная дисфункция как центральное звено патогенеза хронических болезней. Казанский медицинский журнал, 96(4): 659-665 [Mel'nikova Yu.S., Makarova T.P. (2015) Endothelial dysfunction as the key link of chronic diseases pathogenesis. Kazan Medical Journal [Kazanskij medicinskij zhurnal], 96(4): 659-665 (in Russian)]

Момот А.П. (2011) Современные методы распознавания состояния тромботической готовности. Барнаул, 138 с. [Momot А.P. (2011) Modern methods of thrombotic readiness detection. Barnaul, 138 p. (in Russian)]

Рыжков С.В., Полонская Е.И., Заболотняя Е.В., Жилина Е.Б., Алехина М.А., Курбатова Э.В., Курбатов М.Г., Демидова А.А. (2014) Клиническая значимость проведения тромбоэластографии в практике акушера-гинеколога. Международный журнал прикладных и фундаментальных исследований, 12: 101-104 [Ryzhkov S.V., Polonskaja E.I., Zabolotnjaja E.V., Zhilina E.B., Alehina M.A., Kurbatova J.V., Kurbatov M.G., Demidova A.A. (2014) Clinical significance of thromboelastography in practice an obstetrician-gynecologist. International Journal of Applied and Fundamental Research [Mezhdunarodnyj zhurnal prikladnyh i fundamental'nyh issledovanij], 12: 101-104 (in Russian)]

Пшенникова М.Г. (2001) Феномен стресса. Эмоциональный стресс и его роль в патологии. Патологическая физиология и экспериментальная терапия, 3: 28-40 [Pshennikova M.G. (2001) Stress phenomenon. Emotional stress and its role in pathology. Pathological Physiology and Experimental Therapy [Patologicheskaya fiziologiya i eksperimental'naya terapiya], 3: 28-40 (in Russian)]

Шахматов И.И. (2010) Влияние различной продолжительности однократной физической нагрузки и иммобилизации на реакции системы гемостаза. Фундаментальные исследования, 3: 144-150 [Shakhmatov I.I. (2010) Single physical exercises and immobilization of different duration impact haemostatic system reactions. Fundamental Research [Fundamental'nye issledovaniya], 3: 144-150 (in Russian)]

Fras Z., Keber D., Chandler W.L. (2004) The effect of submaximal exercise on fibrinolysis. Blood Coagulation and Fibrinolysis, 15(3): 227-234

Hanke A.A., Staib A., Gorlinger K., Perrey M., Dirkmann D., Kienbaum P. (2010) Whole blood coagulation and platelet activation in the athlete: a comparison of marathon, triathlon and long distance cycling. European Journal of Medical Research, 15(2): 59-65

Johansson P.I., Svendsen M.S., Salado J., Bochsen L., Kristensen A.T. (2008) Investigation of the thrombin-generating capacity, evaluated by thrombogram, and clot formation evaluated by 
thrombelastography of platelets stored in the blood bank for up to 7 days. Vox Sanguinis, 94(2): $113-118$

Johansson P.I., Stissing T., Bochsen L., Ostrowski S.R. (2009) Thromboelastography and thromboelastometry in assessing coagulopathy in trauma. Scandinavian Journal of Trauma, Resuscitation and Emergency Medicine, 17: 45

Kawasaki J., Katori N., Kodaka M., Miyao H., Tanaka K.A. (2004) Electron microscopic evaluation of clot morphology during thrombelastography. Anesthesia and Analgesia, 99(5): 1440-1444

Marijon E., Uy-Evanado A., Reinier K., Teodorescu C., Narayanan K., Jouven X., Gunson K., Jui J., Chugh S.S. (2015) Sudden cardiac arrest during sports activity in middle age. Circulation, 131(16): 1384-1391

Peat E.E., Dawson M., McKenzie A., Hillis W.S. (2010) The effects of acute dynamic exercise on haemostasis in first class Scottish football referees. British Journal of Sports Medicine, 44(8): 573-578

Peng H.T., Rhind S.G. (2015) Thromboelastographic study of psychophysiological stress: a review. Clinical and Applied Thrombosis-Hemostasis, 21(6): 497-512

Stamler J.S. (1994) Redox signaling: nitrosylation and related target interactions of nitric oxide. Cell, 78(6): 931-936

Sucker C., Zotz R.B., Senft B., Scharf R.E., Kroger K., Erbel R., Mohlenkamp S. (2010) Exerciseinduced hemostatic alterations are detectable by rotation thrombelastography (ROTEM): a marathon study. Clinical and Applied Thrombosis-Hemostasis, 16(5): 543-548

Sumann G., Fries D., Griesmacher A., Falkensammer G., Klingler A., Koller A., Streif W., Greie S., Schobersberger B., Schobersberger W. (2007) Blood coagulation activation and fibrinolysis during a downhill marathon run. Blood Coagulation and Fibrinolysis, 18(5): 435-440

Theusinger O.M., Wanner G.A., Emmert M.Y., Billeter A., Eismon J., Seifert B., Simmen H.P., Spahn D.R., Baulig W. (2011) Hyperfibrinolysis diagnosed by rotational thromboelastometry (ROTEM1) is associated with higher mortality in patients with severe trauma. Anesthesia and Analgesia, 113(5): 1003-1012

Trzebicki J., Kuzminska G., Domagala P. (2009) Thromboelastometry - a new method supporting the therapeutical decisions in the coagulopathy based on the Hartet's thromboelastography. Polski Merkuriusz Lekarski, 27(158): 85-91 